\title{
Motor Learning Interference Is Proportional to Occlusion of LTP-Like Plasticity
}

\author{
Gabriela Cantarero, ${ }^{1,2}$ Byron Tang, ${ }^{1}$ Rebecca 0 'Malley, ${ }^{1}$ Rachel Salas, ${ }^{3}$ and Pablo Celnik ${ }^{1,2,3}$ \\ ${ }^{1}$ Department of Physical Medicine and Rehabilitation, Johns Hopkins Medical Institution, Baltimore, Maryland 21205, ${ }^{2}$ Department of Neuroscience, \\ School of Medicine, Johns Hopkins University, Baltimore, Maryland 21205, and ${ }^{3}$ Department of Neurology, Johns Hopkins Outpatient Center, Baltimore, \\ Maryland 21287
}

Learning interference occurs when learning something new causes forgetting of an older memory (retrograde interference) or when learning a new task disrupts learning of a second subsequent task (anterograde interference). This phenomenon, described in cognitive, sensory, and motor domains, limits our ability to learn multiple tasks in close succession. It has been suggested that the source of interference is competition of neural resources, although the neuronal mechanisms are unknown. Learning induces long-term potentiation (LTP), which can ultimately limit the ability to induce further LTP, a phenomenon known as occlusion. In humans we quantified the magnitude of occlusion of anodal transcranial direct current stimulation-induced increased excitability after learning a skill task as an index of the amount of LTP-like plasticity used. We found that retention of a newly acquired skill, as reflected by performance in the second day of practice, is proportional to the magnitude of occlusion. Moreover, the degree of behavioral interference was correlated with the magnitude of occlusion. Individuals with larger occlusion after learning the first skill were (1) more resilient to retrograde interference and (2) experienced larger anterograde interference when training a second task, as expressed by decreased performance of the learned skill in the second day of practice. This effect was not observed if sufficient time elapsed between training the two skills and LTP-like occlusion was not present. These findings suggest competition of LTP-like plasticity is a factor that limits the ability to remember multiple tasks trained in close succession.

\section{Introduction}

Memory consolidation is described as the process through which an initially fragile memory becomes transformed into a stable one (Lechner et al., 1999; Robertson et al., 2004; Krakauer and Shadmehr, 2006). This notion was originally described when researchers found that memory for a list of words was disrupted if new information was learned immediately after, but not if learned 10 min later (Lechner et al., 1999). This phenomenon has also been described in other domains, like sensory perception (Jump and Ries, 2008; Ries and DiGiovanni, 2009) and motor control (Brashers-Krug et al., 1996; Shadmehr and BrashersKrug, 1997; Goedert and Willingham, 2002; Walker et al., 2003; Krakauer et al., 2005; Criscimagna-Hemminger and Shadmehr, 2008). The disruption of the first task memory by a second task has been named retrograde interference. Interestingly, a second form of interference, called anterograde interference, has also been described where training of a task can disrupt subsequent

Received Oct. 4, 2012; revised Nov. 21, 2012; accepted Dec. 22, 2012.

Author contributions: G.C. and P.C. designed research; G.C., B.T., R.O., and R.S. performed research; G.C. and R.O. analyzed data; G.C. and P.C. wrote the paper.

This work was supported by the National Institute of Child Health and Development-National Institutes of Health (Grant R01HD040289), the Brain Science Institute of Johns Hopkins University, and the National Institute of Neurological Disorders and Stroke (Grant 1F31NS073386-01A1). We thank Amy Bastian for critical comments on earlier versions of this manuscript.

Correspondence should be addressed to Pablo Celnik, Department of Physical Medicine and Rehabilitation, Johns Hopkins Medical Institution, 707 North Broadway, Baltimore, MD 21205. E-mail: pcelnik@jhmi.edu.

DOI:10.1523/JNEUROSCI.4706-12.2013

Copyright $\odot 2013$ the authors $\quad 0270-6474 / 13 / 334634-08 \$ 15.00 / 0$ acquisition and/or retention of a second task. Importantly, if sufficient time passes between training of the first and second task, both forms of interference decrease (Brashers-Krug et al., 1996; Shadmehr and Brashers-Krug, 1997; Walker et al., 2003; Krakauer et al., 2005; Criscimagna-Hemminger and Shadmehr, 2008).

Although interference has been well characterized behaviorally, it remains poorly understood why it happens. In animal studies, motor learning leads to long-term potentiation (LTP) in the primary motor cortex (M1). This learning-induced LTP also results in a reduced capacity to induce more LTP. This phenomenon, described as occlusion of LTP induction (Rioult-Pedotti et al., 1998, 2000, 2007; Monfils and Teskey, 2004; Hodgson et al., 2005), can last hours/days before the capacity of neurons to undergo LTP is restored (Rioult-Pedotti et al., 2007). Although it has been speculated that the temporary failure to induce synaptic plasticity in the motor cortex is the physiological signature of interference in motor learning (Rioult-Pedotti et al., 2000; Martin and Morris, 2001), evidence supporting this conjecture is lacking. In humans, evidence of LTP and its occlusion can be probed indirectly using noninvasive brain stimulation techniques able to induce LTP-like plasticity changes. Similar to animal studies, prior investigations in humans showed that motor learning potentiates M1 and results in a temporary occlusion of further potentiation (Ziemann et al., 2004; Stefan et al., 2006; Rosenkranz et al., 2007).

Here, we used anodal transcranial direct current stimulation (AtDCS), a form of noninvasive brain stimulation known to increase 


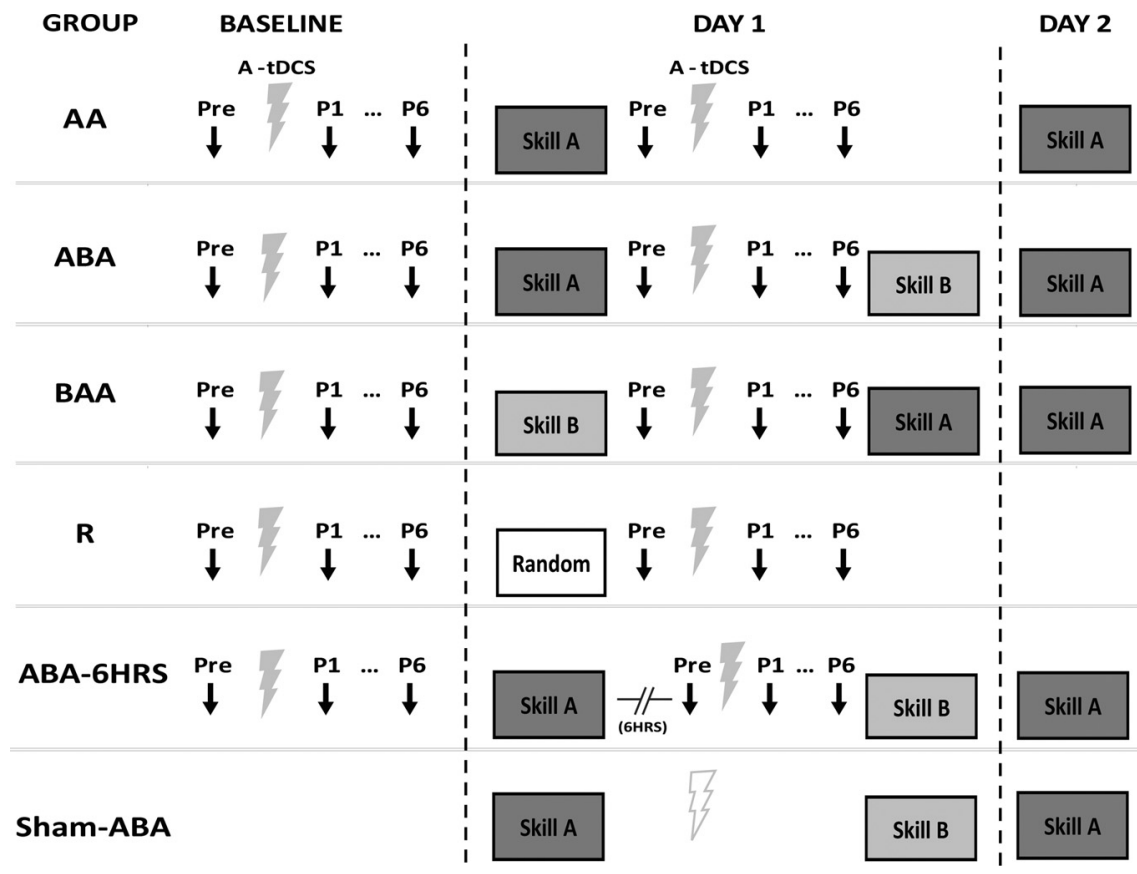

Figure 1. Experimental protocol for all groups. Group AA $(n=11)$ trained Skill $A$, and then returned the following day for a retention test of Skill A. Group ABA ( $n=10)$ trained Skill A, then immediately trained Skill B, then returned the following day for a retention test. Group BAA $(n=10)$ trained Skill $B$ first then Skill A, and returned the following day for a retention test. Group $R$ $(n=10)$ trained a randomized version of the skill task. Training involved practice of each of the skill tasks in four epochs of 30 trials. Group ABA-6HRS $(n=8)$ trained Skill A and B identical to Group ABA except that a $6 \mathrm{~h}$ delay was introduced on the first day between training of Skill $A$ and $B$. In these groups, MEP amplitudes (black arrows) were measured before and after application of A-tDCS (gray ray). This was assessed on separate days, DO when there was no training and on D1 after training. To control for the effects of A-tDCS in between training blocks Group Sham-ABA $(n=8)$ trained on Skills A and B, as done in Group ABA, but with sham tDCS (light gray ray) applied in between training.

excitability via NMDA receptor modulation (Liebetanz et al., 2002, Fritsch et al., 2010; Nitsche et al., 2003), to investigate whether occlusion of LTP-like aftereffects is associated with learning interference. Importantly, interference can be described in terms of disruption of acquisition and/or disruption of retention. Given that previous work showed that M1 plays a central role in retention of motor learning (Muellbacher et al., 2002; Richardson et al., 2006; Hadipour-Niktarash et al., 2007; Reis et al., 2009; Galea et al., 2011), we hypothesized that occlusion of LTP-like plasticity in M1 would correlate with resilience to retrograde interference and increased anterograde interference of retention when subjects learn two consecutive skills.

\section{Materials and Methods}

The study was approved by the Johns Hopkins School of Medicine Institutional Review Board in accordance to the declaration of Helsinki and written informed consent was obtained from all participants. The experiment was performed on 60 healthy subjects ( 27 men, 30 women, ranging from ages 18-37) with no history of neurological disorders. However, three subjects were eliminated due to technical failures during the experimental protocol.

\section{Behavioral measurements}

Skill task: sequential visual isometric pinch task. The generalities of the skill task have been previously described (Reis et al., 2009). Subjects were seated in front of a computer monitor and held an isometric force transducer between the thumb and index finger of the dominant hand. Pinching the force transducer controlled the movement of an on-screen cursor. Participants were instructed to navigate the cursor as quickly and accurately as possible between a HOME position and five gates by alternating the pinch force exerted onto the transducer. The sequence is HOME-1-HOME-2-HOME-3-HOME-4-HOME-5. For this study, we used three variations of the task. In the first variation of the task, we used a logarithmic transduction of pinch force onto cursor movement (Skill A) (as done in Reis et al., 2009). In the second variation, the device had an exponential transduction of pinch force onto cursor movement (Skill B). To control for effort and performance without accumulative learning, the third variation used a randomized force transducer-visual display mapping from trial to trial (Randomized Task). We defined the movement time per trial as movement onset to reaching gate five. We calculated the error rate as the proportion of trials with at least one over or under shooting movement per epoch, where each epoch was the average of 30 consecutive trials.

To quantify motor learning we determined changes in the speed-accuracy trade-off function (SAF) for Skill A. The proposed estimate of changes in the SAF is the skill measure, $a$, as follows:

$$
a=\frac{1-\text { error rage }}{\text { error rate }\left(\text { In }(\text { movement time })^{b}\right)},
$$

where error rate and movement time are averages over 30 trials, and the value of $b$ is 5.424 (Reis et al., 2009). Of note, we performed a similar analysis for Skill B and found that changes in SAF for Skill B were also captured using the same formula.

Training and follow-up. Participants were randomly assigned to one of four groups. Group AA $(n=11)$ subjects trained 120 trials (4 epochs of 30 trials) of Skill A, then returned the following day for a retention test of Skill A (120 trials; Fig. 1). Group ABA $(n=10)$ was the retrograde interference group where subjects trained 120 trials of Skill A and then immediately afterward trained 120 trials of Skill B. These subjects returned the following day for a retention test (120 trials) of Skill A. Group BAA $(n=10)$ was the anterograde interference group where subjects trained 120 trials of Skill B immediately followed by 120 trials of Skill A, and then returned the following day for a retention test of Skill A (120 trials). To control the effects of mere performance rather than learning, Group R $(n=10)$ subjects trained 120 trials of the randomized version of the skill task where accumulation of learning was not expected.

Subsequently, we assessed two additional control groups. To corroborate the association between physiological changes and behavior, we tested Group ABA-6HRS $(n=8)$ to assess whether the typical timedependent reduction in interference is accompanied by a reduction of occlusion of plasticity. Here, subjects trained 120 trials of Skill A followed by a $6 \mathrm{~h}$ break and then trained 120 trials of Skill B. These subjects returned the following day for a retention test of Skill A (120 trials). Finally, to determine whether tDCS between training influenced behavior, a group of subjects trained on Skills A and B, as done in Group ABA, but with sham $\mathrm{tDCS}$ applied in between training (Sham-ABA; $n=8$ ).

To assess retrograde interference we compared performance of Skill A in day 2 relative to day 1 when Skill A was learned in isolation (AA) versus when Skill A was learned before Skill B (ABA). To determine anterograde interference we contrasted the performance of Skill $A$ in day 2 relative to day 1 when Skill A was learned after Skill B (BAA) relative to learning Skill $A$ in isolation (AA). In this manner, we always measured and compared learning of Skill A, whether the training occurred in isolation, or before or after training Skill B.

To measure performance of Skill A we calculated: (1) online gains, defined as the skill measure difference between the last and first epoch for each day and (2) Day 2 relative to Day 1 difference (D2-D1), determined as the overall $\mathrm{D} 2$ performance (mean skill measure for all 
epochs of D2) minus the overall D1 performance (mean skill measure for all epochs of D2).

\section{Neurophysiological measurements}

In addition to training, each subject underwent physiological measurements to assess changes in corticomotor excitability before and after A-tDCS. We measured excitability by quantifying motor-evoked potential amplitudes (MEP) elicited with transcranial magnetic stimulation (TMS). This procedure was done both while subjects were at rest (Day 0, D0) and after training the first behavioral task on D1 (Fig. 1).

Recording. Electromyographic (EMG) activity was recorded using disposable surface electrodes placed over the first dorsal interosseous (FDI) muscle of the dominant hand. Signals were sampled at $2 \mathrm{kHz}$, visually displayed online, and analyzed off-line using MATLAB (MathWorks).

$t D C S$. This was applied via two conducting $25 \mathrm{~cm}^{2}$ electrodes covered with saline-soaked sponges. Using a Phoresor II Auto device (model PM850; OMED), we implemented a bipolar electrode montage (contralateral M1 and ipsilateral supra-orbital area). In this manner we applied A-tDCS for 7 min over the corticomotor hand representation of the FDI muscle, as identified by TMS, at an intensity of $1 \mathrm{~mA}$. This form of stimulation has been shown to increase cortical excitability through NMDA receptor, GABA, BDNF, and calcium-dependent mechanisms in animals/humans (Islam et al., 1995; Liebetanz et al., 2002; Nitsche et al., 2003; Stagg et al., 2009; Fritsch et al., 2010) and induce LTP in mice slice preparations (Fritsch et al., 2010; Ranieri et al., 2012). Importantly, we have performed a preliminary assessment and determined that $7 \mathrm{~min}$ of A-tDCS resulted in increased excitability aftereffects that return to baseline within $30 \mathrm{~min}$. Thus, $7 \mathrm{~min}$ of stimulation was chosen to ensure that by the time subjects began training the second skill task, the aftereffects of A-tDCS had dissipated.

Corticomotor excitability measures. We performed TMS using a flat figure-eight-shaped magnetic coil connected to a Magstim $200^{2}$ magnetic stimulator. Using a frameless neuronavigation system (BrainSight; Rogue Research) we first coregistered subjects' heads to a standard magnetic resonance image. We then identified and marked as the "hot spot" the optimal area of the primary motor cortex (M1) for eliciting MEPs in the resting FDI muscle. In this location, we determined the resting motor threshold as the minimum TMS intensity that evoked an MEP of $50 \mu \mathrm{V}$ in at least 5 of 10 trials in the resting FDI muscle (Rossini et al., 1994). Muscle relaxation was monitored by visual feedback of the EMG recording.

To assess corticomotor excitability we first determined the stimulus intensity needed to evoke an MEP with peak-to-peak amplitude of $\sim 1$ $\mathrm{mV}$ (Stimulus Intensity $1 \mathrm{mV}, \mathrm{S} 1 \mathrm{mV}$ ). Then, $10 \mathrm{MEPs}$ were recorded before and after A-tDCS using the same $\mathrm{S} 1 \mathrm{mV}$ intensity.

Quantification of LTP-like plasticity (index of occlusion). To assess the baseline effect of A-tDCS on cortical excitability, S1 mV was used to record MEP amplitudes before and after application of A-tDCS on a separate day, when subjects were at rest the entire session (D0). To assess the duration of the tDCS aftereffects, $\mathrm{S} 1 \mathrm{mV}$ was repeated every $5 \mathrm{~min}$ for 25 min following A-tDCS (P1, P2, .., P6; Fig. 1). For each subject the average of $10 \mathrm{MEP}$ amplitudes for each time point was normalized to the average of $10 \mathrm{MEP}$ amplitudes before application of A-tDCS. Changes in MEP amplitudes were expressed as a ratio relative to the pre-tDCS MEP amplitude.

On D1 following training, we performed identical measurements of MEP amplitudes before and after application of A-tDCS. In addition, before $\mathrm{TDCS}$ and after training we adjusted the TMS intensity to elicit an MEP of $\sim 1 \mathrm{mV}$. We did this to allow a direct comparison of potentiation plasticity effects elicited by tDCS when applied at rest or after skill train- ing. We used the same protocol to measure MEP amplitude and assess A-tDCS aftereffects in the ABA-6HRS group; however, these were tested $6 \mathrm{~h}$ following training of the first skill. In the Sham-ABA group, the A-tDCS was ramped up to $1 \mathrm{~mA}$ and then immediately ramped down so that subjects felt an initial sensation, but received no more than $30 \mathrm{~s}$ of stimulation.

To quantify the magnitude of occlusion of potentiation plasticity for each subject, we compared the changes induced by A-tDCS on D0 (baseline day) and D1 (training day). The peak MEP amplitude following application of $\mathrm{tDCS}$ was normalized to the MEP amplitude before tDCS for each individual subject. This measurement was done for both D0 and D1. To this end, we developed an occlusion index (OI) for each subject with the following formula:

$$
O I=\left[\frac{\text { Post MEP }}{\text { Pre MEP }}\right]_{\mathrm{D} 0}-\left[\frac{\text { Post MEP }}{\text { Pre MEP }}\right]_{\mathrm{DI}} .
$$

This measurement was used as an index of how much plasticity was used during training, where larger values for the OI are indicative of more occlusion, which would imply more resources were used to induce plasticity changes during learning.

\section{Data analysis}

We used separate polynomial nested repeated measures of ANOVA $\left(\mathrm{ANOVA}_{\mathrm{RM}}\right)$ for the different measures. Post hoc analysis was done with two-tailed $t$ tests when appropriate.

The primary behavioral outcome measure was the skill measure. Differences in performance of Skill A were compared using ANOVA RM $_{\text {with }}$ GROUP (AA, ABA, BAA, R) as the between-factor, and DAY (D1, D2) and EPOCH (Epoch1, Epoch2, Epoch3, Epoch4) as within-factors.

The subsequently added ABA-6HRS control group was assessed similarly comparing performance of Skill A using ANOVA RM $_{\text {with GROUP }}$ (AA, ABA-6HRS) as the between-factor, and DAY (D1, D2) and EPOCH (Epoch1, Epoch2, Epoch3, Epoch4) as within-factors. Finally, the Sham$\mathrm{ABA}$ group was compared with subjects recruited in the ABA group using ANOVA $_{\mathrm{RM}}$ with GROUP (ABA, Sham-ABA) as the between-factor, and DAY (D1, D2) and EPOCH (Epoch1, Epoch2, Epoch3, Epoch4) as within-factors.

The primary outcome measure for corticomotor excitability was the peak-to-peak MEP amplitudes. The amount of potentiation plasticity aftereffects was compared using ANOVA $_{\mathrm{RM}}$ with factors GROUP (AA, $\mathrm{ABA}, \mathrm{BAA}, \mathrm{R})$ as the between-factor, and DAY (D0, D1) and TIME (pre-tDCS, and mean of $[\mathrm{P} 1, \mathrm{P} 2, \mathrm{P} 3, \mathrm{P} 4, \mathrm{P} 5, \mathrm{P} 6]$ ) as within-factors. Similarly, we compared the potentiation plasticity aftereffects in the 

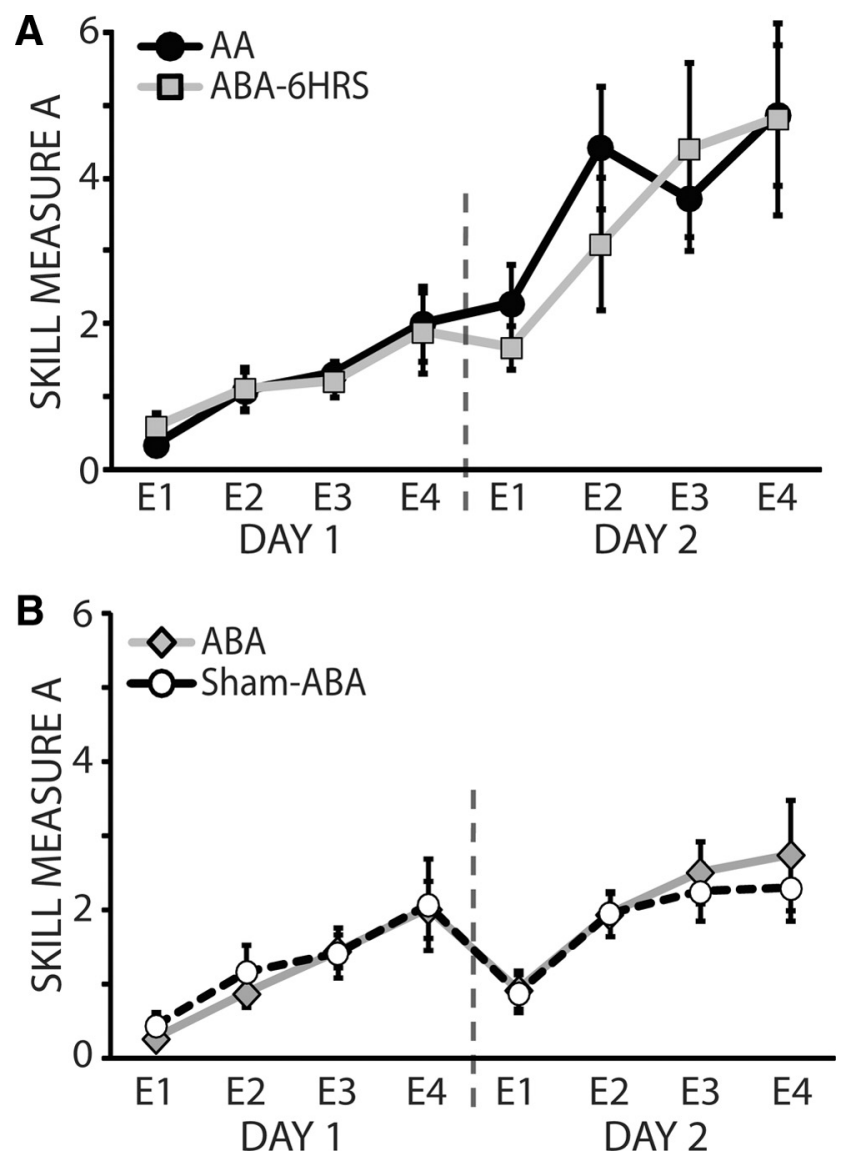

Figure 3. Behavioral results for the ABA-6HRS and sham stimulation control groups. $\boldsymbol{A}$, Performance of Skill A for the ABA-6HRS group. The $y$-axis represents the skill measure of $A$ and the $x$-axis depicts epochs of training. Black circles are the average performances of Skill $A$ in the AA group; light gray squares represent Skill A in the ABA-6HRS group. Vertical dotted line denotes the separation between D1 and D2 of training. Subjects in the ABA-6HRS group showed similar performance of Skill $A$ on both D1 and D2 of training compared with subjects who trained in Group AA. $B$, Performance of Skill A for the Sham-ABA group. The $y$-axis represents the skill measure of $A$ and the $x$-axis depicts epochs of training. Black circles are the average performances of Skill A in the ABA group and light gray diamonds represent Skill A in the Sham-ABA group. Vertical dotted line denotes the separation between D1 and D2 of training. Note that subjects who trained in the Group ABA with real stimulation showed similar performance and interference compared with subjects in Group ABA with sham stimulation. Data are means \pm SEM.

ABA-6HRS control group across DAY (D0, D1) and TIME (pre-tDCS, and mean of [P1, P2, P3, P4, P5, P6]).

To determine associations between OI and behavior we performed a separate correlation analysis using Spearman's $\rho$ for Groups AA, ABA, and BAA. Our a priori hypothesis was that there would be a difference between the BAA and ABA correlations. To test this, we first performed the Fisher $z$ transformation to normalize the underlying distribution of each correlation, $r_{1}$ and $r_{2}$ (Zar, 1999, equation 19.8):

$$
z=0.5 \operatorname{In}\left(\frac{1+r}{1-r}\right) .
$$

The normalized correlation coefficients allowed us to calculate a $z$-score (Zar, 1999, equation 19.21) with ABA group as Group A and BAA group as Group B as follows:

$$
\mathrm{Z}=\frac{\mathrm{Z}_{\mathrm{A}}-\mathrm{Z}_{\mathrm{B}}}{\sqrt{\frac{1}{n_{A}-3}+\frac{1}{n_{B}-3}}} .
$$

All statistical analyses were performed using SPSS. All hypothesis tests are reported as two-tailed. Effects were considered significant if $p \leq 0.05$. All data are given as means \pm SEM.

\section{Results}

Behavioral interference in motor skill learning

Subjects who trained in either the ABA or BAA groups experienced behavioral interference, as evidenced by poorer performance on the second day of practice compared with subjects who trained Skill A alone in the AA group (AA:ABA, $p=0.02$; AA: BAA, $p=0.03$; Fig. 2A). Online (within-day) learning was not different between groups, whereas performance on D2 relative to D1 (D2-D1) was significantly better in the Group AA relative to the $\mathrm{ABA}(p<0.01)$ and BAA $(p=0.01)$ groups (Fig. $2 B)$. These results indicate that behavioral differences between Group AA and the interference groups were not due to differences in the skill measure on D1, but were specific to performance on D2. In other words, retention across days of Skill A, as reflected by performance in D2, was interfered in both the anterograde and retrograde groups. In addition, subjects that trained in Group $\mathrm{R}$ showed no improvement in performance.

Of note, it would seem logical to suggest that individuals who occluded the most after learning Skill A would have the poorest acquisition of Skill B, thus minimizing any sort of retrograde interference on Skill A because Skill B was never learned to begin with. However, we found a significant correlation where subjects who acquired Skill A the best also acquired Skill B the best and vice versa $\left(R^{2}=0.55 ; p<0.01\right)$. This indicates that the interference observed on D2 in the BAA group was not due to a lack of acquisition of Skill A on D1.

In the additional controls, we found that subjects training in the ABA-6HRS group showed similar performance relative to Group AA (Fig. 3A). Consistent with prior studies (BrashersKrug et al., 1996; Shadmehr and Brashers-Krug, 1997; Muellbacher et al., 2002; Walker et al., 2003; Krakauer et al., 2005; Robertson et al., 2005; Robertson, 2012), this indicates that waiting $6 \mathrm{~h}$ between training Skill A and B reduces the interference observed in the ABA and BAA groups. Finally, participants in the Sham-ABA group showed similar performance as subjects in the ABA group (Fig. $3 B$ ), indicating that A-tDCS applied in between skill training did not affect performance or interference.

\section{Error versus movement time}

Given the seemingly odd result that the anterograde interference group experienced interference of skill retention but not acquisition (lack of interference at D1), we dissected the skill measure into its components (error and movement time) to assess whether these parameters were differentially affected (Fig. 4). We found that subjects who trained in the BAA group had significantly more errors on Skill A on D1 compared with both Group $\mathrm{AA}(p=0.02)$ and Group ABA $(p=0.03$; Fig. $4 A)$. However, on $\mathrm{D} 1$, subjects in the BAA group were also significantly faster on Skill A compared with Group AA $(p=0.05)$ and marginally faster than Group ABA ( $p=0.07$; Fig. $4 B$ ). Importantly, these differences were not present on D2.

These results indicate that on D1 subjects who trained in the BAA group experienced interference when assessing errors, but gained in performance when measuring movement speed. Since skill improvement is the consequence of changes in both movement time and accuracy (Reis et al., 2009), the net skill gain in D1 for the BAA group was similar to the ABA and AA groups. 
Occlusion of LTP-like plasticity

following motor skill learning

MEPs on D0 in the AA, ABA, BAA, and R groups were significantly larger following application of A-tDCS $(p<0.01, p=$ $0.05, p=0.05, p=0.01$, respectively; Fig. 5). However, the same A-tDCS protocol failed to increase MEPs when applied after training on $\mathrm{D} 1$ in the $\mathrm{AA}, \mathrm{ABA}$, and $\mathrm{BAA}$ groups. Specifically, the mean MEP amplitudes after A-tDCS in the baseline day (D0) were significantly larger than in the training day (D1) for the AA ( $p=0.02)$, $\operatorname{ABA}(p=0.02)$, and $\operatorname{BAA}(p=0.04)$ groups. This indicates that skill training occluded A-tDCS aftereffects on excitability. In contrast, subjects training on the randomized task (Group R) showed similar increases in MEP amplitudes on both D0 and D1 after application of A-tDCS

(D0: pre-tDCS vs post-tDCS: $p=0.01$; 1 : pre-tDCS vs posttDCS $p<0.01)$, but no difference in the amount of tDCS aftereffects when comparing post tDCS effects across days ( $p=0.47$; Fig. 5). These results suggest that the occlusion of A-tDCSinduced increase in excitability was specifically observed during learning, but not after practice where learning does not occur or accumulate.

Similarly, subjects that trained in the ABA-6HRS group (where the A-tDCS protocol was introduced $6 \mathrm{~h}$ following training of Skill A) showed comparable increases in MEP amplitudes on both $\mathrm{D} 0$ and $\mathrm{D} 1$, indicating no difference in the magnitude of tDCS aftereffects (D0: pre-tDCS vs post-tDCS: $p<0.01$; D1: pre-tDCS vs post-tDCS $p=0.02$; Fig. 6 ).

Altogether these results show that after learning either Skill A or Skill B, subjects had a significant reduction of the typical enhancement of excitability expected from the application of A-tDCS. This type of physiological response (lack of facilitation to a stimulation protocol following learning) has been characterized as occlusion of LTP in animal studies (Rioult-Pedotti et al., 1998, 2007; Monfils and Teskey, 2004; Hodgson et al., 2005;) and has been described in previous investigations in humans (Ziemann et al., 2004; Stefan et al., 2006; Rosenkranz et al., 2007). Importantly, when we assessed excitability changes $6 \mathrm{~h}$ after training the magnitude of A-tDCS aftereffects was similar to D0 indicating that LTP-like capacity was restored $6 \mathrm{~h}$ following learning of the skill task.

\section{Behavioral-physiological correlation of motor skill learning}

We predicted that larger occlusion effects (suggestive of more LTP-like plasticity resources used) after learning one skill would be associated with better performance of the skill the following day as well as more resilience to retrograde interference. Furthermore, the same plasticity change should lead to more anterograde interference, as indicated by decreased skill performance on D2. We conducted a correlation between behavior (the difference in performance between D2 and D1) and physiological changes (the OI). We found a significant correlation between the OI and the skill performance difference between practice days for Groups $\mathrm{AA}\left(R^{2}=0.74 ; p<0.01\right), \mathrm{ABA}\left(R^{2}=0.57 ; p<0.01\right)$, and $\mathrm{BAA}$ $\left(R^{2}=0.72 ; p<0.01 ;\right.$ Fig. 7). Specifically, AA subjects with larger amounts of occlusion after training Skill A showed better performance of Skill A the following day. Similarly, ABA subjects with larger amounts of occlusion after training Skill A were more re-

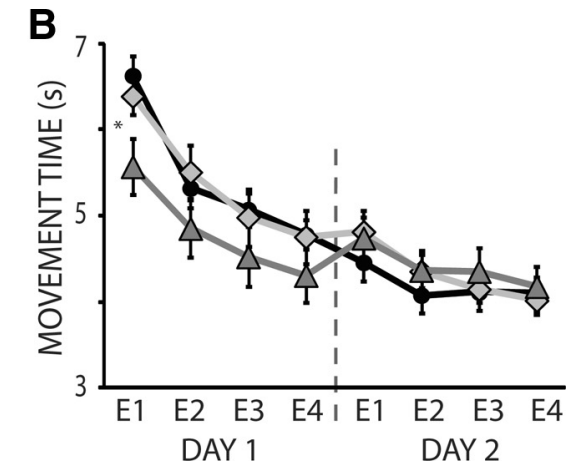

Figure 4. Error versus movement time. Black circles are the average performances of Skill A in the AA group, light gray diamonds represent Skill A in the ABA group, dark gray triangles are the Skill A performance average in the BAA group. Vertical dotted line otes the separation between D1 and D2 of training. $A$, The $y$-axis represents the error rate of Skill $A$ and the $x$-axis shows epochs . Subjects who trained in the BAA paradigm had significantly more errors in Skill A on D1 compared with both Group AA and $A B A$. $B$, The $y$-axis represents the movement time of $A$ and the $x$-axis depicts subsequent epochs of training. Subjects who

silient to retrograde interference from Skill B on performance of Skill A the following day. On the other hand, BAA subjects that showed larger amounts of occlusion following training of Skill B experienced more anterograde interference effects on the performance of Skill A the following day. Importantly, these two correlations (ABA and BAA) were significantly different from each another $(p<0.01)$. Altogether, these results indicate an association between occlusion of A-tDCS aftereffects and skill performance in a subsequent day (AA group), as well as between occlusion and behavioral interference.

Importantly, testing a correlation between the tDCS response at baseline (nontraining day) and behavioral performance (D2D1) showed a trend for subjects training in the AA group $\left(R^{2}=\right.$ $0.41 ; p=0.07)$. However, there was no relationship between baseline tDCS aftereffects and interference in learning. In other words, although baseline response to tDCS may be associated with the ability to learn one skill, it is not possible to predict how this response might influence learning multiple skills in close succession (learning interference).

\section{Discussion}

The main results of this study show that when individuals learn a new motor skill, the magnitude of occlusion of A-tDCS aftereffects (reflective of how much LTP-like plasticity resources were used for learning) was associated with better performance the following day. Moreover, the occlusion observed after learning a motor skill was positively correlated with anterograde interference and negatively correlated with retrograde interference, as depicted by performance on the second day. Importantly, introducing a $6 \mathrm{~h}$ delay following training of the first task restored LTP-like capacity and reduced behavioral interference. Altogether, these results indicate that competition for resources involved in LTP-like plasticity is a mechanism underlying learning interference.

Animal and human studies have shown that motor learning is associated with LTP/LTP-like plasticity in M1. This effect is associated with a reduced capacity to sustain further LTP (RioultPedotti et al., 1998, 2000, 2007; Monfils and Teskey, 2004; Hodgson et al., 2005) or LTP-like plasticity in humans (Ziemann et al., 2004; Stefan et al., 2006; Rosenkranz et al., 2007), and an increased capacity for long-term depression (Monfils and Teskey, 2004; Rioult-Pedotti et al., 2007). This is thought to be mediated by a "synaptic modification range" within which syn- 


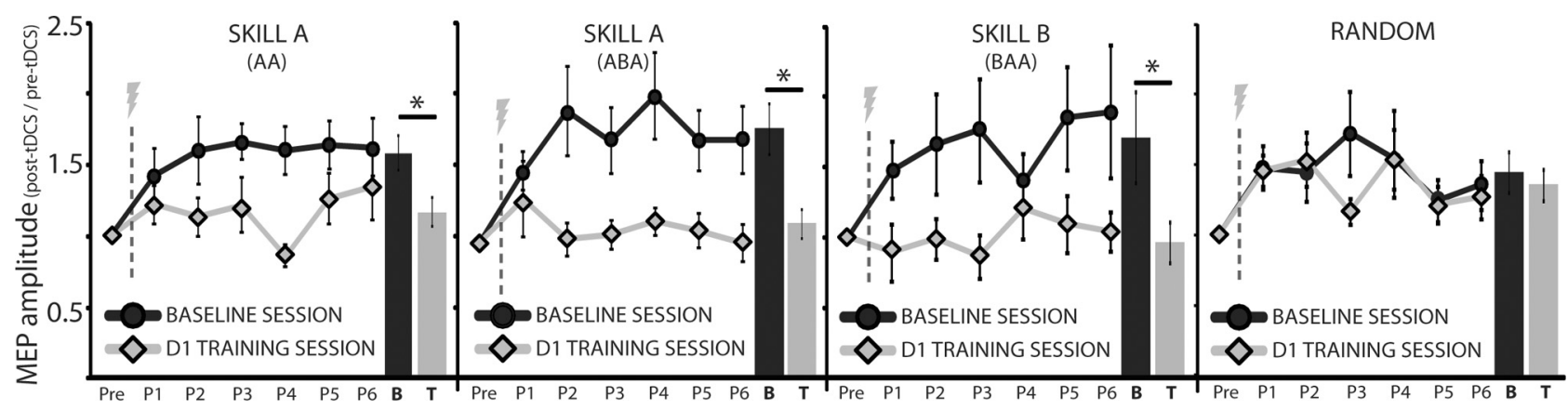

Figure 5. MEP amplitude ratios for pre- and post-A-tDCS. The $y$-axis represents the average MEP amplitude standardized to the pre-tDCS MEP amplitude and the $x$-axis represents successive TMS measurements taken before application of A-tDCS (Pre), immediately after A-tDCS (Post 1; P1) and repeated every 5 min up to 25 min post-A-tDCS (P2...P6). Black circles are the mean MEP amplitude for all subjects on D0 (Baseline Day). Gray diamonds are the mean MEP amplitude for all subjects on D1 (Training Day). Bar graphs depict average MEP amplitude for P1-P6 for Baseline Session (B) and for Training Session (T). Far left graph is MEP amplitudes for AA subjects after training Skill A. Second from left graph is MEP amplitudes for ABA subjects after training Skill A. Second from right graph is MEP amplitudes after training Skill B. Far right graph is the mean MEP amplitude for subjects in Group R (no learning). After training either Skill A or Skill B, subjects had a significant reduction of potentiation aftereffects following application of A-tDCS (occlusion) compared with following a period of rest. Subjects who trained on the randomized task showed similar amounts of LTP-like plasticity either at rest or following training demonstrating no occlusion when accumulation of learning does not occur. Data are means \pm SEM. ${ }^{*} p \leq 0.05$.

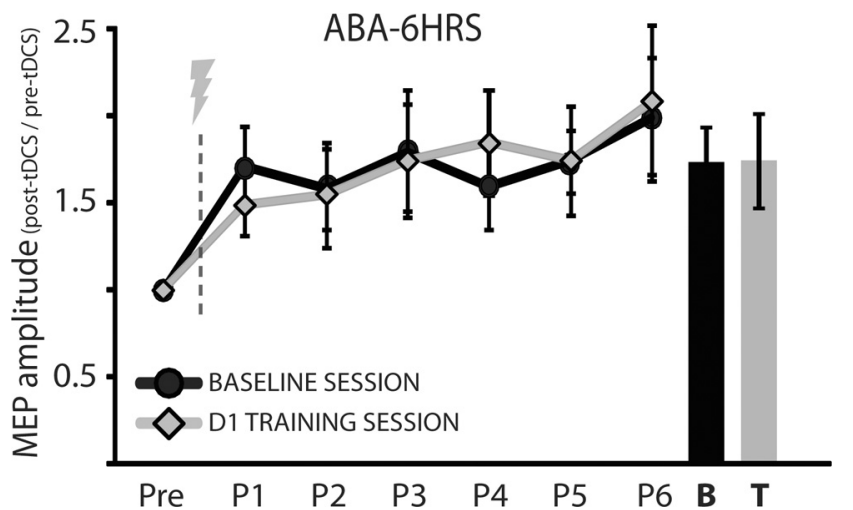

Figure 6. MEP amplitude ratios for pre- and post-A-tDCS of the ABA-6HRS control group. The $y$-axis represents the average MEP amplitude standardized to the pre-tDCS MEP amplitude and the $x$-axis represents successive TMS measurements taken before application of A-tDCS (Pre), immediately after A-tDCS (Post 1,P1), and repeated every 5 min up to 25 min post-A-tDCS (P2...P6). Black circles are the mean MEP amplitude for all subjects on DO (Baseline Day). Gray diamonds are the mean MEP amplitude for all subjects on D1 (Training Day). Bar graph depicts average MEP amplitude for P1-P6 for Baseline Session (B) and for Training Session (T). Note that MEP measurements were assessed $6 \mathrm{~h}$ following training of Skill A. Data are means \pm SEM.

aptic efficacy can be modified. Any attempts to either potentiate or depress neuronal synapses beyond this range result in no additional changes in excitability (Rioult-Pedotti et al., 1998, 2000, 2007; Martin and Morris, 2001; Stefan et al., 2006). The implication of this phenomenon is that learning shifts synaptic efficacy upward within the synaptic modification range and "uses up" some of the potentiating plasticity available for further retention of learning. Thus, it has been speculated that the temporary failure to induce LTP-like plasticity in M1 could be a physiological marker of interference in motor learning (Rioult-Pedotti et al., 2000; Martin and Morris, 2001).

Our findings suggest that competition over limited capacity to induce changes in LTP-like plasticity leads to a reduced ability to retain multiple tasks trained in close succession. Importantly, this is not to argue that a limited capacity to undergo LTP-like plasticity changes is the only mechanism that underlies retention and interference. Although our study focused on LTP-like plasticity changes, likely other forms of plasticity are also involved such as modulation of facilitatory and inhibitory networks and structural modifications. In fact, animal studies have shown correlations between morphological change in M1 and behavior (Xu et al., 2009).

\section{Speed versus accuracy}

Previous investigations addressing motor learning interference have used adaptation or skill tasks focusing on only one behavioral component, either speed or accuracy. Here, we studied skill as a whole, assessing changes in the SAF as well as in its individual components. By doing this, we found an interesting effect: anterograde interference when evaluating overall skill was only evident in the second day of training rather than immediately after training the first skill. This finding, previously unrecognized, can be explained by a trade-off of error (accuracy) versus movement time (speed) for the benefit of overall skill performance.

Subjects in the BAA group made significantly more errors during the Skill A acquisition period of D1, but had faster movement times relative to subjects in the AA and ABA groups. Thus, if one were to clamp one behavioral component (as is generally done in motor learning studies), the interpretation that emerges becomes drastically different. For instance, in studies using dynamic and kinematic adaptation tasks (Brashers-Krug et al., 1996; Shadmehr and Brashers-Krug 1997; Wigmore et al., 2002; Krakauer et al., 2005; Criscimagna-Hemminger and Shadmehr, 2008), subjects were restricted to move within a fixed movement time and only changes in accuracy were assessed as the behavioral outcome measure. In those studies (when task A and task B were learned in close succession), accuracy was impaired during acquisition of the second task. This is consistent with the current study, where subjects who trained Skill B before training Skill A showed impairment in accuracy. Thus if the results of the BAA group were interpreted based solely on errors, then the results would look similar to the classic example of anterograde interference. However, if the behavior had been interpreted solely in terms of movement time (Goedert et al., 2002; Walker et al., 2003, references), the conclusion would be the exact opposite, where training on Skill B actually transferred some benefit to subsequent acquisition of task $\mathrm{A}$.

Though unexpected, our findings have important implications. They indicate that training two motor skills in close succession can result in both positive transfer of speed and negative transfer of accuracy. Generalization of motor learning has been defined as the ability to apply what has been learned in one context to other contexts. When generalization is beneficial, it is 
termed transfer, and when it is detrimental, it is termed interference (Krakauer and Shadmehr, 2006). Although, motor control studies typically view interference and transfer of learning as very different and independent phenomena, the present results suggest that perhaps the two are intimately related.

\section{Effect of A-tDCS on behavior}

Previous work has shown that application of A-tDCS over M1 during training of a motor task can lead to improved performance (Reis et al., 2009; Fritsch et al., 2010). Importantly, these and other studies have suggested that performance enhancement does not occur if A-tDCS is not applied simultaneously with training, such as before (Kuo et al., 2008), after (Fritsch et al., 2010), or in between (Reis et al., 2009) training sessions. In this study, to avoid potential overlaps between the aftereffects of A-tDCS and behavior, we chose $7 \mathrm{~min}$ of A-tDCS because in prior unpublished observations by our group we found that the aftereffects of 7 min of A-tDCS lasted $30 \mathrm{~min}$. In this way we ensured that by the time subjects began training on their second skill task, the aftereffects of A-tDCS had dissipated. Nonetheless, it may still be possible that A-tDCS between training of the first and second motor skills could have influenced the interference process. To address this potential confound, we studied the effect of sham tDCS stimulation in the ABA schedule (Sham-ABA). This group showed similar behavioral effects as the A-tDCS ABA group, indicating that A-tDCS between skill training did not affect the magnitude of learning interference.

\section{Limitations}

It is possible that the experimental design used in this study affected the magnitude of behavioral interference. For instance, the time delay necessary to test the physiological measurements between training of Skills A and B may have diminished the interference effect size. Despite this, the training of the second task still occurred within the consolidation time window, as evidenced by poorer performance in the subsequent day in the ABA and BAA groups. In addition, it is possible that a night of sleep before the retention test may have offered an opportunity to salvage motor memories, again potentially reducing the magnitude of the interference effects. However, subjects still showed a clear performance impairment the following day, indicating that interference had occurred. In addition, due to the lack of focality intrinsic to the use of $\mathrm{A}-\mathrm{tDCS}$, it is not possible to determine whether occlusion of A- $\mathrm{tDCS}$ effects after motor learning depict homosynaptic or heterosynaptic mechanisms.

\section{Summary and conclusions}

In sum, our results indicate that the physiological phenomenon of occlusion of LTP-like plasticity is associated with three important behavioral correlates: (1) the more occlusion (indicative of more potentiation resource being used), the better performance and more resilience to retrograde interference from a second task on a subsequent day, suggesting a retention mechanism; (2) the more occlusion, the more interference with the performance of a second behavioral skill task the following day, suggesting that the phenomenon of anterograde interference is due to retention of one skill interfering with the retention of a second skill; and (3) if there is a sufficient time delay that allows restoration of LTP-like mechanisms, there is no behavioral interference. Interestingly, the relationship between plasticity occlusion mechanisms and behavioral skill improvement are specifically related to motor learning, given that the lack of performance improvement in the random group was associated with no plasticity occlusion. In addition, our findings cannot be explained by the application of A-tDCS in between training tasks because applying sham tDCS elicited the same amount of learning interference as in the real stimulation group.

The present results have broad practical implications for skill learning practice schedules, and raise an important question: Can we use a measure of LTP-like plasticity occlusion as a biomarker to predict the amount of retention and interference (i.e., impairment in retention) when training new tasks? Perhaps there is a limitation in how much the motor cortex can retain in a day/ session before its resources to sustain potentiating plasticity are exhausted, beyond this point increased retention becomes negligible. This also opens the opportunity to test interventions to reset the system to improve retention and reduce interference. Finally, it is important to note that although not tested here, similar plasticity competition mechanisms may be the physiological substrate of learning interference in other cognitive (Lechner et al., 1999) and sensory (Jump et al., 2008; Ries and DiGiovanni, 2009) domains.

\section{References}

Brashers-Krug T, Shadmehr R, Bizzi E (1996) Consolidation in human motor memory. Nature 382:252-255. CrossRef Medline

Criscimagna-Hemminger SE, Shadmehr R (2008) Consolidation patterns of human motor memory. J Neurosci 28:9610-9618. CrossRef Medline Fritsch B, Reis J, Martinowich K, Schambra HM, Ji Y, Cohen LG, Lu B (2010) 
Direct current stimulation promotes BDNF-dependent synaptic plasticity: potential implications for motor learning. Neuron 66:198-204. CrossRef Medline

Galea JM, Vazquez A, Pasricha N, de Xivry JJ, Celnik P (2011) Dissociating the roles of the cerebellum and motor cortex during adaptive learning: the motor cortex retains what the cerebellum learns. Cereb Cortex 21:1761-1770. CrossRef Medline

Goedert KM, Willingham DB (2002) Patterns of interference in sequence learning and prism adaptation inconsistent with the consolidation hypothesis. Learn Mem 9:279-292. CrossRef Medline

Hadipour-Niktarash A, Lee CK, Desmond JE, Shadmehr R (2007) Impairment of retention but not acquisition of a visuomotor skill through time-dependent disruption of primary motor cortex. J Neurosci 27:13413-13419. CrossRef Medline

Hodgson RA, Ji Z, Standish S, Boyd-Hodgson TE, Henderson AK, Racine RJ (2005) Training-induced and electrically induced potentiation in the neocortex. Neurobiol Learn Mem 83:22-32. CrossRef Medline

Islam N, Aftabuddin M, Moriwaki A, Hattori Y, Hori Y (1995) Increase in the calcium level following anodal polarization in the rat brain. Brain Res 684:206-208. CrossRef Medline

Jump RL, Ries DT (2008) Effect of interaural level and phase cues on intervening interference in auditory working memory for loudness. Hear Res 240:112-115. CrossRef Medline

Krakauer JW, Shadmehr R (2006) Consolidation of motor memory. Trends Neurosci 29:58-64. CrossRef Medline

Krakauer JW, Ghez C, Ghilardi MF (2005) Adaptation to visuomotor transformations: consolidation, interference, and forgetting. J Neurosci 25: 473-478. CrossRef Medline

Kuo M-F, Unger M, Liebetanz D, Lang N, Tergau F, Paulus W, Nitsche MA (2008) Limited impact of homeostatic plasticity on motor learning in humans. Neuropsychologia 46:2122-2128. CrossRef Medline

Lechner HA, Squire LR, Byrne JH (1999) 100 years of consolidation-remembering Müller and Pilzecker. Learn Mem 6:77-87. Medline

Liebetanz D, Nitsche MA, Tergau F, Paulus W (2002) Pharmacological approach to the mechanisms of transcranial DC-stimulation-induced aftereffects of human motor cortex excitability. Brain 125:2238-2247. CrossRef Medline

Martin SJ, Morris RG (2001) Cortical plasticity: it's all the range!. Curr Biol 11:R57-R59. Medline

Monfils MH, Teskey GC (2004) Skilled-learning-induced potentiation in rat sensorimotor cortex: a transient form of behavioural long-term potentiation. Neuroscience 125:329-336. CrossRef Medline

Muellbacher W, Ziemann U, Wissel J, Dang N, Kofler M, Facchini S, Boroojerdi B, Poewe W, Hallett M (2002) Early consolidation in human primary motor cortex. Nature 415:640-644. CrossRef Medline

Nitsche MA, Fricke K, Henschke U, Schlitterlau A, Liebetanz D, Lang N, Henning S, Tergau F, Paulus W (2003) Pharmacological modulation of cortical excitability shifts induced by transcranial direct current stimulation in humans. J Physiol 553:293-301. CrossRef Medline

Ranieri F, Podda MV, Riccardi E, Frisullo G, Dileone M, Profice P, Pilato F, Di Lazzaro V, Grassi C (2012) Modulation of LTP at rat hippocampal CA3-CA1 synapses by direct current stimulation. J Neurophysiol 107:1868-1880. CrossRef Medline

Reis J, Schambra HM, Cohen LG, Buch ER, Fritsch B, Zarahn E, Celnik PA, Krakauer JW (2009) Noninvasive cortical stimulation enhances motor skill acquisition over multiple days through an effect on consolidation. Proc Natl Acad Sci U S A 106:1590-1595. CrossRef Medline
Richardson AG, Overduin SA, Valero-Cabré A, Padoa-Schioppa C, PascualLeone A, Bizzi E, Press DZ (2006) Disruption of primary motor cortex before learning impairs memory of movement dynamics. J Neurosci 26 : 12466-12470. CrossRef Medline

Ries DT, DiGiovanni JJ (2009) Effects of recurrent tonal information on auditory working memory for pitch. Hear Res 255:14-21. CrossRef Medline

Rioult-Pedotti MS, Friedman D, Hess G, Donoghue JP (1998) Strengthening of horizontal cortical connections following skill learning. Nat Neurosci 1:230-234. CrossRef Medline

Rioult-Pedotti MS, Friedman D, Donoghue JP (2000) Learning-induced LTP in neocortex. Science 290:533-536. CrossRef Medline

Rioult-Pedotti MS, Donoghue JP, Dunaevsky A (2007) Plasticity of the synaptic modification range. J Neurophysiol 98:3688-3695. CrossRef Medline

Robertson EM (2012) New insights in human memory interference and consolidation. Curr Biol 22:R66-R71. CrossRef Medline

Robertson EM, Pascual-Leone A, Miall RC (2004) Current concepts in procedural consolidation. Nat Rev Neurosci 5:576-582. CrossRef Medline

Robertson EM, Press DZ, Pascual-Leone A (2005) Off-line learning and the primary motor cortex. J Neurosci 25:6372-6378. CrossRef Medline

Rosenkranz K, Kacar A, Rothwell JC (2007) Differential modulation of motor cortical plasticity and excitability in early and late phases of human motor learning. J Neurosci 27:12058-12066. CrossRef Medline

Rossini PM, Barker AT, Berardelli A, Caramia MD, Caruso G, Cracco RQ, Dimitrijević MR, Hallett M, Katayama Y, Lücking CH, et al. (1994) Non-invasive electrical and magnetic stimulation of the brain, spinal cord and roots: basic principles and procedures for routine clinical application. Report of an IFCN committee. Electroencephalogr Clin Neurophysiol 91:79-92. CrossRef Medline

Shadmehr R, Brashers-Krug T (1997) Functional stages in the formation of human long-term motor memory. J Neurosci 17:409-419. Medline

Stagg CJ, Best JG, Stephenson MC, O'Shea J, Wylezinska M, Kincses ZT, Morris PG, Matthews PM, Johansen-Berg H (2009) Polarity-sensitive modulation of cortical neurotransmitters by transcranial stimulation. J Neurosci 29:5202-5206. CrossRef Medline

Stefan K, Wycislo M, Gentner R, Schramm A, Naumann M, Reiners K, Classen J (2006) Temporary occlusion of associative motor cortical plasticity by prior dynamic motor training. Cereb Cortex 16:376-385. Medline

Walker MP, Brakefield T, Hobson JA, Stickgold R (2003) Dissociable stages of human memory consolidation and reconsolidation. Nature 425:616-620. CrossRef Medline

Wigmore V, Tong C, Flanagan JR (2002) Visuomotor rotations of varying size and direction compete for a single internal model in motor working memory. J Exp Psychol Hum Percept Perform 28:447-457. CrossRef Medline

Xu T, Yu X, Perlik AJ, Tobin WF, Zweig JA, Tennant K, Jones T, Zuo Y (2009) Rapid formation and selective stabilization of synapses for enduring motor memories. Nature 462:915-919. CrossRef Medline

Zar JH (1999) Biostatistical analysis, Ed 4. Upper Saddle River, NJ: Prentice Hall.

Ziemann U, Ilić TV, Pauli C, Meintzschel F, Ruge D (2004) Learning modifies subsequent induction of long-term potentiation-like and long-term depression-like plasticity in human motor cortex. J Neurosci 24:16661672. CrossRef Medline 